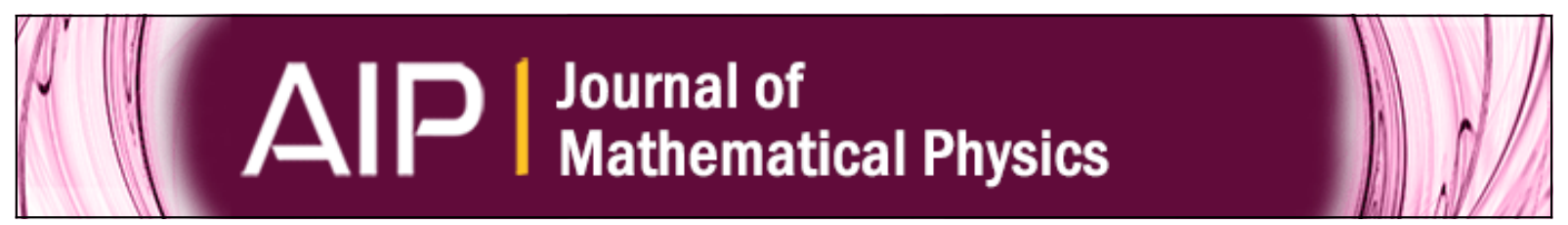

\title{
Analytic structure of the multichannel Jost matrix for potentials with Coulombic tails
}

S. A. Rakityansky and N. Elander

Citation: Journal of Mathematical Physics 54, 122112 (2013); doi: 10.1063/1.4853855

View online: http://dx.doi.org/10.1063/1.4853855

View Table of Contents: http://scitation.aip.org/content/aip/journal/jmp/54/12?ver=pdfcov

Published by the AIP Publishing

\section{Articles you may be interested in}

The functional integral with unconditional Wiener measure for anharmonic oscillator

J. Math. Phys. 49, 113505 (2008); 10.1063/1.3013803

On the Numerical Solution of the TimeDependent Schrödinger Equation with TimeDependent Potentials

AIP Conf. Proc. 1048, 1011 (2008); 10.1063/1.2990863

Analytic Coulomb matrix elements in the lowest Landau level in disk geometry

J. Math. Phys. 43, 1664 (2002); 10.1063/1.1446244

On solvable potentials related to $\mathrm{SO}(2,2)$

J. Math. Phys. 42, 543 (2001); 10.1063/1.1336512

Padé approximant algorithm for solving nonlinear ordinary differential equation boundary value problems on an unbounded domain

Comput. Phys. 11, 299 (1997); 10.1063/1.168606

\section{AlP Re-register for Table of Content Alerts}




\title{
Analytic structure of the multichannel Jost matrix for potentials with Coulombic tails
}

\author{
S. A. Rakityansky ${ }^{1, a)}$ and N. Elander ${ }^{2}$ \\ ${ }^{1}$ Department of Physics, University of Pretoria, Pretoria 0002, South Africa \\ ${ }^{2}$ Division of Chemical Physics, Department of Physics, Stockholm University, \\ Stockholm, SE-106 91, Sweden
}

(Received 28 May 2013; accepted 8 December 2013; published online 31 December 2013)

A quantum system is considered that can move in $N$ two-body channels with the potentials that may include the Coulomb interaction. For this system, the Jost matrix is constructed in such a way that all its dependencies on the channel momenta and Sommerfeld parameters are factorized in the form of explicit analytic expressions. It is shown that the two remaining unknown matrices are single-valued analytic functions of the energy and therefore can be expanded in the Taylor series near an arbitrary point within the domain of their analyticity. It is derived a system of firstorder differential equations whose solutions determine the expansion coefficients of these series. Alternatively, the unknown expansion coefficients can be used as fitting parameters for parametrizing experimental data similarly to the effective-range expansion. Such a parametrization has the advantage of preserving proper analytic structure of the Jost matrix and can be done not only near the threshold energies, but around any collision or even complex energy. As soon as the parameters are obtained, the Jost matrix (and therefore the $S$-matrix) is known analytically on all sheets of the Riemann surface, and thus enables one to locate possible resonances. (c) 2013 AIP Publishing LLC. [http://dx.doi.org/10.1063/1.4853855]

\section{INTRODUCTION}

The use of analytic functions is a common feature of the majority of physics theories. The knowledge of the general structure of these functions, such as the topology of the Riemann surface on which they are defined, the distribution of their singularities, etc., is of utmost importance for an adequate description of the observed phenomena. In the scattering theory, such knowledge enables one to deduce certain general properties of the physical system even without solving the dynamical equations. ${ }^{1}$

The $S$-matrix of the scattering theory is not a single-valued function of the energy variable $E$ even in the simplest case of a single-channel problem with a short-range potential. This is because it depends on the energy via the corresponding momentum $k= \pm \sqrt{2 \mu E / \hbar^{2}}$, which has two possible values (with \pm ) for each point $E$. As a result, the single-channel $S(E)$ is a double-valued function and therefore is defined on a double-sheet Riemann surface with a square-root branching at the threshold energy $E=0$. Furthermore, it is a meromorphic function of $E$, i.e., it is analytic everywhere except for isolated poles corresponding to the spectral points (bound states and resonances). The bound and resonant state poles are on the physical and non-physical sheets of the Riemann surface, respectively. ${ }^{2}$

For a single-channel problem with short-range forces, the "troublesome" dependence of the $S$-matrix on the momentum, that makes it double-valued, can be easily isolated. As it is shown, for example, in Ref. 3, in such a case the partial-wave $S$-matrix is

$$
S_{\ell}(E)=\frac{A_{\ell}(E)+i k^{2 \ell+1} B_{\ell}(E)}{A_{\ell}(E)-i k^{2 \ell+1} B_{\ell}(E)},
$$

\footnotetext{
a)E-mail: rakitsa@up.ac.za
} 
where $A_{\ell}(E)$ and $B_{\ell}(E)$ are single-valued analytic (i.e., entire) functions of $E$, defined on a single complex plane. All the trouble with the branching stems from the factors $k^{2 \ell+1}$ that are odd powers of $k$.

Knowing that $A_{\ell}(E)$ and $B_{\ell}(E)$ are smooth analytic functions, one can approximate them by a finite number of terms of the Taylor series. This is widely used, for example, in the form of the so called effective-range expansion, where the ratio $A_{\ell}(E) / B_{\ell}(E)=k^{2 \ell+1} \cot \delta_{\ell}$ is expanded near the point $E=0$ and the expansion coefficients serve as the free parameters to fit experimental data. Apparently, the expansions of $A_{\ell}(E)$ and $B_{\ell}(E)$ and a similar fitting procedure can be used not only near the threshold, but near any collision energy or even anywhere in the complex $E$-plane and, thanks to correct analytic structure of the $S$-matrix (1), its proper branching is always preserved.

For the $N$-channel problems, the topology of the Riemann surface becomes more and more complicated with the growth of the number of channels $N$ (see Ref. 4). Indeed, the $S$-matrix depends on all the channel momenta

$$
k_{n}= \pm \sqrt{\frac{2 \mu_{n}}{\hbar^{2}}\left(E-E_{n}\right)}, \quad n=1,2, \ldots, N,
$$

which are determined by the differences between the total energy $E$ and the channel thresholds $E_{n}$, as well as by the corresponding reduced masses $\mu_{n}$. There are $2^{N}$ possible combinations of the signs in front of the $N$ square roots (2). As a result, the $S$-matrix is defined on the Riemann surface consisting of $2^{N}$ sheets with a square-root branching at each threshold energy. The intricate topology of the (most simple) two-channel Riemann surface is schematically illustrated in Fig. 1.

In the past, much effort was spent to generalize the effective-range expansion as well as to derive something similar to Eq. (1) for the multi-channel problems. Most of the authors ${ }^{5-10}$ tried to keep as much similarity with the traditional effective-range expansion as possible, i.e., to use the constructions involving $\cot \delta$. As a result, their approaches are rather complicated and, strictly speaking, only applicable for real energies because the scattering phase shift $\delta(E)$ has no meaning outside the real axis. An analog of the expression (1) for the two-channel problem was obtained in Ref. 11 and is known as the Dalitz-Tuan representation (see also Refs. 4 and 12). However, when $N \geq 3$, within the traditional approach based on the $K$-matrix, it becomes extremely difficult to obtain the $S$-matrix with correct branching at all the thresholds. ${ }^{4,13}$

As was emphasized in Ref. 14, the Jost matrix has a much more simpler analytic structure than the corresponding $S$-matrix. Indeed, in Eq. (1), for example, the numerator and denominator are the Jost functions taken with different signs of the momentum. In contrast to the $S$-matrix, they do not have poles (only zeros). If we expand the functions $A_{\ell}(E)$ and $B_{\ell}(E)$ in the Taylor series, the corresponding expansion for the $S$-matrix will be of the Padé type, which is known to have a wider range of validity. When constructing the generalized effective-range expansions, it is therefore much more convenient to deal with the Jost matrices rather than with the $S$-matrix or with something

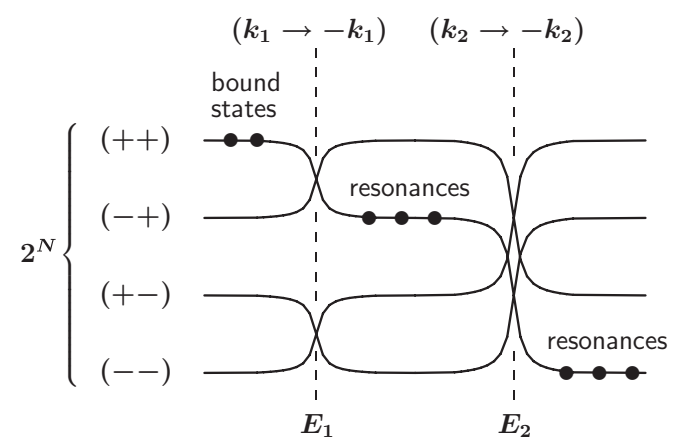

FIG. 1. Interconnections of the Riemann energy-sheets at the thresholds $E_{1}$ and $E_{2}$ for a two-channel $(N=2)$ problem. The sheets $( \pm \pm)$ correspond to all possible combinations of the signs of $\operatorname{Im} k_{n}$. Each interconnection is shown for a full circle path around a threshold. On such a path the corresponding channel momentum changes its sign. The dots schematically show typical distribution of the spectral points. 
that directly involves the phase-shifts. Earlier, similar ideas were suggested by Pupyshev ${ }^{15}$ who developed a perturbation theory based on the Maclaurin expansions of the functions closely related to the Jost functions.

Recently, within an alternative approach based on differential equations for the Jost matrix, a direct generalization was obtained of the representation (1) for an arbitrary number of channels with short-range interactions. ${ }^{16}$ The multi-channel $S$-matrix,

$$
S(E)=f^{(\text {out })}(E)\left[f^{(\text {in })}(E)\right]^{-1},
$$

is the "ratio" of the Jost matrices whose matrix elements have the following structure $(m, n=1,2$, $\ldots, N)$ :

$$
f_{m n}^{(\text {in } / \text { out })}(E)=\frac{k_{n}^{\ell_{n}+1}}{k_{m}^{\ell_{m}+1}} A_{m n}(E) \mp i k_{m}^{\ell_{m}} k_{n}^{\ell_{n}+1} B_{m n}(E),
$$

where $\ell_{n}$ are the channel angular-momenta and the energy dependent matrices $A(E)$ and $B(E)$ are the entire functions of $E$, i.e., all the trouble with the branching is isolated in the momentum-dependent coefficients. This representation was successfully used to fit two-channel scattering data at the energies far away from the thresholds and thus to recover the parameters of the nearby resonances. ${ }^{17}$

In the present paper, we further generalize the representations (3) and (4) by considering the systems of charged particles, i.e., we assume that in addition to the short-range part, the potential may include a Coulomb tail. For the sake of clarity, we first modify the single-channel representation (1) by adding the Coulomb potential, and then do the same for an arbitrary number of channels.

\section{SINGLE-CHANNEL PROBLEM}

Consider a single channel problem for a particle of mass $\mu$ moving in a spherically symmetric potential that is a sum of a short-range and a Coulomb terms. In general, any potential with a Coulombic tail can be written as such a sum by adding and subtracting an appropriate pure Coulomb potential.

After standard separation of the angular dependence, the radial Schrödinger equation reads

$$
\left[\partial_{r}^{2}+k^{2}-\frac{\ell(\ell+1)}{r^{2}}-V(r)-\frac{2 k \eta}{r}\right] u_{\ell}(E, r)=0,
$$

where $k=\sqrt{2 \mu E / \hbar^{2}}$ is the momentum corresponding to the energy $E, \ell$ is the angular momentum, $V(r)$ is the short-range part of the potential, and

$$
\eta=\frac{\mu e^{2} Z_{1} Z_{2}}{k \hbar^{2}}
$$

is the Sommerfeld parameter corresponding to the electric force between two particles of the charges $e Z_{1}$ and $e Z_{2}$.

\section{A. Jost functions}

When $r \rightarrow \infty$, the potential $V(r)$ vanishes and the general solution of Eq. (5) becomes a linear combination of the regular and irregular Coulomb functions $F_{\ell}(\eta, k r)$ and $G_{\ell}(\eta, r)$. Keeping this in mind, we look for $u_{\ell}(E, r)$ as their linear combination at all distances,

$$
u_{\ell}(E, r)=F_{\ell}(\eta, k r) A_{\ell}(E, r)+G_{\ell}(\eta, k r) B_{\ell}(E, r),
$$

where the unknown functions $A_{\ell}(E, r)$ and $B_{\ell}(E, r)$ become $r$-independent at large distances (beyond the range of the potential $V$ ). Actually, this way of solving differential equations is known in mathematics as the variation of parameters method. ${ }^{18}$ Substituting the ansatz (7) in Eq. (5), we obtain an equivalent system of first-order differential equations (the details can be found in Refs. 19 and 20) for the unknown functions $A_{\ell}(E, r)$ and $B_{\ell}(E, r)$,

$$
\partial_{r} A_{\ell}=\frac{1}{k} G_{\ell} V\left(F_{\ell} A_{\ell}+G_{\ell} B_{\ell}\right),
$$




$$
\partial_{r} B_{\ell}=-\frac{1}{k} F_{\ell} V\left(F_{\ell} A_{\ell}+G_{\ell} B_{\ell}\right)
$$

where we dropped the variable symbols $(E, r)$ for the sake of clarity. The boundary conditions for these equations follow from the fact that $u_{\ell}(E, r)$ is the physical wave function and therefore must be regular at $r=0$. The simplest choice that guarantees its regularity is

$$
A_{\ell}(E, 0)=1, \quad B_{\ell}(E, 0)=0 .
$$

In principle, for $A_{\ell}(E, 0)$ we can use any $E$-dependent value. However, as it will be seen later, we need $E$-independent boundary conditions in order to use the Poincarè theorem.

Therefore, near the origin, our solution behaves exactly like the regular Coulomb function,

$$
u_{\ell}(E, r) \underset{r \rightarrow 0}{\longrightarrow} F_{\ell}(\eta, k r) \underset{r \rightarrow 0}{\longrightarrow} C_{\ell}(\eta)(k r)^{\ell+1},
$$

where

$$
C_{\ell}(\eta)=\frac{2^{\ell} e^{-\pi \eta / 2}}{\Gamma(2 \ell+2)}|\Gamma(\ell+1 \pm i \eta)|
$$

is the Coulomb barrier factor. In order to define the Jost function, we need another solution known as the regular solution ${ }^{2}$ and behaving near the origin exactly like the Riccati-Bessel function $j_{\ell}(k r)$,

$$
\phi_{\ell}(E, r) \underset{r \rightarrow 0}{\longrightarrow} j_{\ell}(k r) \underset{r \rightarrow 0}{\longrightarrow} \frac{(k r)^{\ell+1}}{(2 \ell+1) ! !} .
$$

Comparing (11) and (13), we see that $u_{\ell}$ and $\phi_{\ell}$ differ by an energy dependent factor,

$$
\phi_{\ell}(E, r)=\frac{1}{C_{\ell}(\eta)(2 \ell+1) ! !} u_{\ell}(E, r) .
$$

The Jost functions $f_{\ell}^{(\text {in) }}(E)$ and $f_{\ell}^{(\text {out })}(E)$ are the amplitudes of the incoming and outgoing spherical waves in the asymptotic behaviour of the regular solution (see, for example, Ref. 2),

$$
\phi_{\ell}(E, r) \underset{r \rightarrow \infty}{\longrightarrow} h_{\ell}^{(-)}(k r) e^{\eta \ln 2 k r} f_{\ell}^{(\text {in })}(E)+h_{\ell}^{(+)}(k r) e^{-\eta \ln 2 k r} f_{\ell}^{(\text {out })}(E),
$$

where

$$
h_{\ell}^{( \pm)}(k r)=j_{\ell}(k r) \pm i y_{\ell}(k r) \underset{r \rightarrow \infty}{\longrightarrow} \mp i \exp \left[ \pm i\left(k r-\frac{\ell \pi}{2}\right)\right]
$$

are the Riccati-Hankel functions, which are the zero-charge limits $(\eta \rightarrow 0)$ of the corresponding Coulomb functions

$$
H_{\ell}^{( \pm)}(\eta, k r)=F_{\ell}(\eta, k r) \mp i G_{\ell}(\eta, k r) \underset{r \rightarrow \infty}{\longrightarrow} \mp i \exp \left\{ \pm i\left[k r-\eta \ln (2 k r)-\frac{\ell \pi}{2}+\sigma_{\ell}\right]\right\},
$$

where

$$
\sigma_{\ell}=\arg \Gamma(\ell+1+i \eta)
$$

is the pure Coulomb phase-shift. Using these Coulomb spherical waves, we can rewrite Eq. (15) as

$$
\phi_{\ell}(E, r) \underset{r \rightarrow \infty}{\longrightarrow} H_{\ell}^{(-)}(\eta, k r) e^{i \sigma_{\ell}} f_{\ell}^{(\mathrm{in})}(E)+H_{\ell}^{(+)}(\eta, k r) e^{-i \sigma_{\ell}} f_{\ell}^{(\text {out })}(E) .
$$

Introducing the linear combinations of the unknown functions $A_{\ell}$ and $B_{\ell}$,

$$
\mathcal{F}_{\ell}^{\text {(in/out) }}(E, r)=\frac{1}{2}\left[A_{\ell}(E, r) \mp i B_{\ell}(E, r)\right],
$$

which become $r$-independent at large distances in the same way as the functions $A_{\ell}$ and $B_{\ell}$ do, we have

$$
\begin{aligned}
u_{\ell}(E, r) & =H_{\ell}^{(-)}(\eta, k r) \mathcal{F}_{\ell}^{(\mathrm{in})}(E, r)+H_{\ell}^{(+)}(\eta, k r) \mathcal{F}_{\ell}^{(\text {out })}(E, r) \\
& \underset{r \rightarrow \infty}{\longrightarrow} H_{\ell}^{(-)}(\eta, k r) \mathcal{F}_{\ell}^{(\text {in })}(E, \infty)+H_{\ell}^{(+)}(\eta, k r) \mathcal{F}_{\ell}^{(\text {out })}(E, \infty),
\end{aligned}
$$


and therefore form Eqs. (14) and (19) it follows that

$$
f_{\ell}^{(\text {in } / \text { out })}(E)=\lim _{r \rightarrow \infty} \frac{e^{\mp i \sigma_{\ell}}}{C_{\ell}(\eta)(2 \ell+1) ! !} \mathcal{F}_{\ell}^{\text {(in/out) }}(E, r) .
$$

The boundary conditions (10) and the definitions (20) give $\mathcal{F}_{\ell}^{\text {(in/out) }}(E, 0)=1 / 2$. In the pure Coulomb case $(V \equiv 0)$ the right-hand sides of Eqs. (8) and (9)) are identical zeros and therefore the asymptotic values of $\mathcal{F}_{\ell}^{\text {(in/out) }}$ are the same as at the origin. This means that the pure Coulomb Jost functions and the $S$-matrix are

$$
\begin{gathered}
f_{\ell}^{\mathrm{c}(\mathrm{in} / \mathrm{out})}(E)=\frac{e^{\mp i \sigma_{\ell}}}{2 C_{\ell}(\eta)(2 \ell+1) ! !}=\frac{e^{\pi \eta / 2}(2 \ell) ! !}{2^{\ell+1} \Gamma(\ell+1 \pm i \eta)}, \\
S_{\ell}^{\mathrm{c}}(E)=\frac{\Gamma(\ell+1+i \eta)}{\Gamma(\ell+1-i \eta)}=e^{2 i \sigma_{\ell}}
\end{gathered}
$$

\section{B. Analytic structure}

In Ref. 21, by Lambert, it was shown that the Coulomb functions can be factorized as follows:

$$
\begin{aligned}
& F_{\ell}(\eta, k r)=D_{\ell}(\eta, k) \tilde{F}_{\ell}(E, r), \\
& G_{\ell}(\eta, k r)=M(\eta) D_{\ell}(\eta, k) \tilde{F}_{\ell}(E, r)+\frac{k}{D_{\ell}(\eta, k)} \tilde{G}_{\ell}(E, r),
\end{aligned}
$$

where $\tilde{F}_{\ell}(E, r)$ and $\tilde{G}_{\ell}(E, r)$ are entire (analytic and single-valued) functions of the energy variable $E$, while the complicated $k$-dependence, that makes the Coulomb functions multi-valued, is explicitly isolated via the factors

$$
D_{\ell}(\eta, k)=C_{\ell}(\eta) k^{\ell+1}, \quad M(\eta)=\frac{2 \eta h(\eta)}{C_{0}^{2}(\eta)}
$$

with

$$
h(\eta)=\frac{1}{2}[\psi(i \eta)+\psi(-i \eta)]-\ln \hat{\eta}, \quad \psi(z)=\frac{\Gamma^{\prime}(z)}{\Gamma(z)}, \quad \hat{\eta}=\frac{\mu e^{2}\left|Z_{1} Z_{2}\right|}{k \hbar^{2}}
$$

Explicit expressions for the functions $\tilde{F}_{\ell}(E, r)$ and $\tilde{G}_{\ell}(E, r)$ are obtained in Ref. 22 and in a compact form are given in Appendix B of Ref. 23.

Using the Lambert representation, (25) and (26), we can re-write the radial wave function (7) as

$$
u_{\ell}=F_{\ell} A_{\ell}+G_{\ell} B_{\ell}=D_{\ell}\left(\tilde{F}_{\ell} \tilde{A}_{\ell}+\tilde{G}_{\ell} \tilde{B}_{\ell}\right)
$$

where the "tilded" unknown functions $\tilde{A}_{\ell}$ and $\tilde{B}_{\ell}$ are defined by the following equations:

$$
\begin{aligned}
& \tilde{A}_{\ell}(E, r)=A_{\ell}(E, r)+M(\eta) B_{\ell}(E, r), \\
& \tilde{B}_{\ell}(E, r)=\frac{k}{D_{\ell}^{2}(\eta, k)} B_{\ell}(E, r) .
\end{aligned}
$$

With the help of Eqs. (25), (26), and (29) the differential equations (8) and (9) take the form

$$
\begin{aligned}
\partial_{r} A_{\ell} & =\left(\frac{M D_{\ell}^{2}}{k} \tilde{F}_{\ell}+\tilde{G}_{\ell}\right) V\left(\tilde{F}_{\ell} \tilde{A}_{\ell}+\tilde{G}_{\ell} \tilde{B}_{\ell}\right), \\
\partial_{r} B_{\ell} & =-\frac{D_{\ell}^{2}}{k} \tilde{F}_{\ell} V\left(\tilde{F}_{\ell} \tilde{A}_{\ell}+\tilde{G}_{\ell} \tilde{B}_{\ell}\right) .
\end{aligned}
$$

Multiplying Eq. (33) by $M$ and adding the result with Eq. (32) and then multiplying Eq. (33) by $k / D_{\ell}^{2}$, we obtain

$$
\partial_{r} \tilde{A}_{\ell}(E, r)=\tilde{G}_{\ell}(E, r) V(r)\left[\tilde{F}_{\ell}(E, r) \tilde{A}_{\ell}(E, r)+\tilde{G}_{\ell}(E, r) \tilde{B}_{\ell}(E, r)\right],
$$




$$
\partial_{r} \tilde{B}_{\ell}(E, r)=-\tilde{F}_{\ell}(E, r) V(r)\left[\tilde{F}_{\ell}(E, r) \tilde{A}_{\ell}(E, r)+\tilde{G}_{\ell}(E, r) \tilde{B}_{\ell}(E, r)\right] .
$$

The most important feature of these new differential equations is the fact that all their coefficients are entire functions of the parameter $E$ for any finite value of $r$. Moreover, the boundary conditions,

$$
\tilde{A}_{\ell}(E, 0)=1, \quad \tilde{B}_{\ell}(E, 0)=0,
$$

that immediately follow from (10) and (30), (31), do not depend on $E$. According to the Poincaré theorem ${ }^{24}$ (see also Ref. 25), the solutions of Eqs. (34) and (35), i.e., the functions $\tilde{A}_{\ell}(E, r)$ and $\tilde{B}_{\ell}(E, r)$, are analytic and single-valued functions of $E$.

What we actually need is the asymptotic values of $\tilde{A}_{\ell}(E, r)$ and $\tilde{B}_{\ell}(E, r)$ at large distances. Indeed, combining Eqs. (20), (22) and (30), (31), we see that the Jost functions (for a short-range plus a Coulomb potential) have the following structure:

$$
f_{\ell}^{\text {(in/out) }}(E)=\frac{e^{\mp i \sigma_{\ell}}}{2 C_{\ell}(\eta)(2 \ell+1) ! !}\left\{\tilde{A}_{\ell}(E, \infty)-\left[\frac{2 \eta h(\eta)}{C_{0}^{2}(\eta)} \pm i\right] C_{\ell}^{2}(\eta) k^{2 \ell+1} \tilde{B}_{\ell}(E, \infty)\right\} .
$$

Taking the limit $r \rightarrow \infty$ in Eqs. (34) and (35) is a delicate procedure. When $E$ is a real scattering energy, everything is simple: both the regular and irregular Coulomb functions on the right-hand sides of these equations are oscillating with finite amplitudes. Thanks to this, the short-range potential $V(r)$ causes the right-hand sides vanish very fast with growing $r$.

However, if $E$ is complex, the Coulomb functions (which can always be written as linear combinations of the functions (17)) become exponentially growing, which may cause the divergence. This difficulty and a way to go around it were discussed in Refs. 16 and 26, where differential equations similar to (34) and (35) were obtained. It was shown that the solutions of these equations at $r \rightarrow \infty$ remain holomorphic functions of $E$ within a domain $\mathcal{D}$ along the positive real axis. The shape and width of $\mathcal{D}$ are determined by the character of $V(r)$. In particular, for an exponentially falling potential $V(r)$, this domain is of a parabolic type, $\operatorname{Re} E \sim(\operatorname{Im} E)^{2}$. In any case, the functions $\tilde{A}_{\ell}(E, \infty)$ and $\tilde{B}_{\ell}(E, \infty)$ can always be analytically continued outside the domain $\mathcal{D}$ by using complex rotation of the coordinate, $r \rightarrow r e^{i \theta}$, which causes the $\mathcal{D}$ to turn as is schematically shown in Fig. 2. We therefore can conclude that in the analytic structure (37), the functions $\tilde{A}_{\ell}(E, \infty)$ and $\tilde{B}_{\ell}(E, \infty)$ are holomorphic (at least) in a wide domain around the positive real axis, which means at all points of practical interest, where Eq. (37) could be used to describe scattering processes and resonances.

\section{Short-range plus attractive Coulomb potential}

In all the derivation throughout the paper, we assume that the charges $Z_{1} e$ and $Z_{2} e$ have arbitrary signs, i.e., the Coulomb potential can be either attractive or repulsive. A pure Coulomb potential with $Z_{1} Z_{2}<0$ always generates the hydrogen-like spectrum of the bound state energies,

$$
E_{n}^{c}=-\frac{\left|Z_{1} Z_{2}\right|^{2} \mu e^{4}}{2 \hbar^{2} n^{2}}
$$

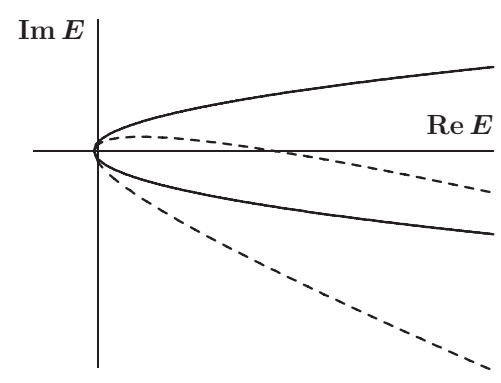

FIG. 2. Schematically shown domain $\mathcal{D}$ (within the parabola) where the functions $\tilde{A}_{\ell}(E, \infty)$ and $\tilde{B}_{\ell}(E, \infty)$ are holomorphic, for an exponentially falling potential $V(r)$. The dashed curve shows what happens to $\mathcal{D}$, when the coordinate is rotated, $r \rightarrow e^{i \theta} r$ with $\theta>0$. 
where the principal quantum number, $n=1,2,3, \ldots$, is composed of the radial quantum number $n_{r}$ $=0,1,2, \ldots$ and the orbital number $\ell=0,1,2, \ldots$,

$$
n=n_{r}+\ell+1 \text {. }
$$

At all these energies, the Sommerfeld parameter is pure imaginary,

$$
\eta_{n}=i n
$$

which makes singular the denominator on the right-hand side of Eq. (23) for the Jost function $f_{\ell}^{\mathrm{c}(\mathrm{in})}(E)$,

$$
\Gamma\left(\ell+1+i \eta_{n}\right)=\Gamma(\ell+1-n)=\Gamma\left(-n_{r}\right)=\infty . \quad n_{r}=0,1,2, \ldots
$$

Therefore, (as is expected) the spectral points (38) correspond to the zeros of $f_{\ell}^{\mathrm{c}(\mathrm{in})}(E)$ and to the poles of the corresponding $S$-matrix (24).

Apparently, if a short-range potential is added to an attractive Coulomb potential, the Coulombic bound-state spectrum is somehow modified. In this connection, the structure of the Jost function $f_{\ell}^{(\mathrm{in})}(E)$, given by Eq. (37), may cause confusion. Indeed, rewriting this equation as

$$
f_{\ell}^{(\mathrm{in})}(E)=f_{\ell}^{\mathrm{c}(\mathrm{in})}(E)\left\{\tilde{A}_{\ell}(E, \infty)-\left[\frac{2 \eta h(\eta)}{C_{0}^{2}(\eta)}+i\right] C_{\ell}^{2}(\eta) k^{2 \ell+1} \tilde{B}_{\ell}(E, \infty)\right\},
$$

one may erroneously conclude that the Jost function $f_{\ell}^{(\mathrm{in})}(E)$ always has zeros at the hydrogen-like energies (38), independently of the choice of the short-range potential, because the pure Coulomb Jost function is a factor in (40). It is however not difficult to see that the other factor in Eq. (40), i.e., the expression in the curly brackets, is singular at the energies (38),

$$
f_{\ell}^{(\text {in })}\left(E_{n}^{c}\right)=0 \times\left\{\tilde{A}_{\ell}-\left[\frac{\infty}{\infty}+i\right] \times \infty \times k_{n}^{2 \ell+1} \tilde{B}_{\ell}\right\} .
$$

In the Appendix, it is shown that these singularities and the zero cancel each other in such a way that the Jost function (40) is finite and generally non-zero at the Coulomb energies (38),

$$
f_{\ell}^{(\mathrm{in})}\left(E_{n}^{c}\right)=0 \times \infty=\mathrm{const}\left\{\begin{array}{l}
\neq 0 \\
\neq \infty
\end{array} .\right.
$$

Of course some of the spectral points of the total potential may accidentally coincide with the pure Coulomb spectral points, and thus $f_{\ell}^{(\text {in) }}\left(E_{n}^{c}\right)=0$ at them. This, however, is only possible if $\tilde{B}_{\ell}\left(E_{n}^{c}, \infty\right)=0$, which in general is not the case.

Therefore, the factorized representation (37) of the Jost functions is valid even when the Coulombic part of the total potential is attractive. However, when using it near the spectral points of the pure Coulomb potential, one should do it with care because the singularities cancel each other at these points. In formal derivations this fact does not pose any problem. But in numerical calculations the cancelation of singularities is always a source of errors. In such a case, it is needed to replace the Jost function with its approximate expression (A11), which is valid near a Coulombic spectral point $E_{n}^{c}$.

\section{Power-series expansions}

The fact that $\tilde{A}_{\ell}(E, r)$ and $\tilde{B}_{\ell}(E, r)$ are holomorphic functions of the energy means that they can be expanded in the Taylor series,

$$
\begin{aligned}
& \tilde{A}_{\ell}(E, r)=\sum_{n=0}^{\infty} \alpha_{n}\left(\ell, E_{0}, r\right)\left(E-E_{0}\right)^{n}, \\
& \tilde{B}_{\ell}(E, r)=\sum_{n=0}^{\infty} \beta_{n}\left(\ell, E_{0}, r\right)\left(E-E_{0}\right)^{n},
\end{aligned}
$$


around an arbitrary point $E_{0}$ within the domain $\mathcal{D}$ of the complex $E$-plane (for a finite $r$, this domain is whole complex plane). In order to find the expansion coefficients, $\alpha_{n}$ and $\beta_{n}$, we do the expansions of the Coulomb functions,

$$
\begin{aligned}
& \tilde{F}_{\ell}(E, r)=\sum_{n=0}^{\infty} \varphi_{n}\left(\ell, E_{0}, r\right)\left(E-E_{0}\right)^{n}, \\
& \tilde{G}_{\ell}(E, r)=\sum_{n=0}^{\infty} \gamma_{n}\left(\ell, E_{0}, r\right)\left(E-E_{0}\right)^{n},
\end{aligned}
$$

around the same point $E_{0}$, and substitute Eqs. (42)-(45) into the differential equations (34) and (35). Equalizing the coefficients of the same powers of $\left(E-E_{0}\right)$, we obtain

$$
\begin{array}{ll}
\partial_{r} \alpha_{n} & =\sum_{i+j+k=n} \gamma_{i} V\left(\varphi_{j} \alpha_{k}+\gamma_{j} \beta_{k}\right), \\
\partial_{r} \beta_{n} & =-\sum_{i+j+k=n} \varphi_{i} V\left(\varphi_{j} \alpha_{k}+\gamma_{j} \beta_{k}\right), \quad n=0,1,2, \ldots,
\end{array}
$$

with simple boundary conditions,

$$
\alpha_{n}\left(\ell, E_{0}, 0\right)=\delta_{n 0}, \quad \beta_{n}\left(\ell, E_{0}, 0\right)=0, \quad \forall n, \ell, E_{0} .
$$

After finding several coefficients $\alpha_{n}\left(\ell, E_{0}, \infty\right)$ and $\beta_{n}\left(\ell, E_{0}, \infty\right)$, we obtain power-series expansions of the Jost functions (37) near a chosen point $E_{0}$, and through them the corresponding approximation of the $S$-matrix (3). Such an expansion can be considered as a generalized effective-range series.

The coefficients $\alpha_{n}$ and $\beta_{n}$ could be obtained either by solving differential equations (46) and (47), or alternatively by fitting some scattering data near $E_{0}$. In the latter case, these coefficients serve as the free parameters. In both approaches, the approximate $S$-matrix has proper analytic structure and is valid near $E_{0}$ on all sheets of the Riemann surface.

\section{Effective-range expansion}

The standard effective-range series can be obtained by putting $E_{0}=0$ and considering real positive (collision) energies $E$, for which it is meaningful to introduce the scattering phase-shift. In such a case, the functions $\tilde{A}_{\ell}(E, \infty)$ and $\tilde{B}_{\ell}(E, \infty)$ are real. This means that the numerator and denominator of the $S$-matrix,

$$
\begin{aligned}
S_{\ell}(E)= & \frac{\tilde{A}_{\ell}-\left(\frac{2 \eta h}{C_{0}^{2}}-i\right) C_{\ell}^{2} k^{2 \ell+1} \tilde{B}_{\ell}}{\tilde{A}_{\ell}-\left(\frac{2 \eta h}{C_{0}^{2}}+i\right) C_{\ell}^{2} k^{2 \ell+1} \tilde{B}_{\ell}} e^{2 i \sigma_{\ell}} \\
= & \frac{\tilde{A}_{\ell}-\left(\frac{2 \eta h}{C_{0}^{2}}-i\right) C_{\ell}^{2} k^{2 \ell+1} \tilde{B}_{\ell}}{\tilde{A}_{\ell}-\left(\frac{2 \eta h}{C_{0}^{2}}+i\right) C_{\ell}^{2} k^{2 \ell+1} \tilde{B}_{\ell}} \cdot \frac{\Gamma(\ell+1+i \eta)}{\Gamma(\ell+1-i \eta)},
\end{aligned}
$$

are complex conjugates to each other, i.e., they have the same absolute value, but the opposite signs of the phase:

$$
\delta_{\ell}^{\mathrm{sc}}=\arg \left[\tilde{A}_{\ell}-\left(\frac{2 \eta h}{C_{0}^{2}}-i\right) C_{\ell}^{2} k^{2 \ell+1} \tilde{B}_{\ell}\right]
$$

This gives

$$
S_{\ell}=e^{2 i \delta_{\ell}^{\mathrm{sc}}} e^{2 i \sigma_{\ell}}
$$


and

$$
\cot \delta_{\ell}^{\mathrm{sc}}=\frac{\operatorname{Re}\left[\tilde{A}_{\ell}-\left(\frac{2 \eta h}{C_{0}^{2}}-i\right) C_{\ell}^{2} k^{2 \ell+1} \tilde{B}_{\ell}\right]}{\operatorname{Im}\left[\tilde{A}_{\ell}-\left(\frac{2 \eta h}{C_{0}^{2}}-i\right) C_{\ell}^{2} k^{2 \ell+1} \tilde{B}_{\ell}\right]}=\frac{\tilde{A}_{\ell}-\frac{2 \eta h}{C_{0}^{2}} C_{\ell}^{2} k^{2 \ell+1} \tilde{B}_{\ell}}{C_{\ell}^{2} k^{2 \ell+1} \tilde{B}_{\ell}} .
$$

Finally, we obtain the standard expansion,

$$
\left(\cot \delta_{\ell}^{\mathrm{sc}}+\frac{2 \eta h}{C_{0}^{2}}\right) C_{\ell}^{2} k^{2 \ell+1}=\frac{\alpha_{0}+\alpha_{1} E+\alpha_{2} E^{2}+\cdots}{\beta_{0}+\beta_{1} E+\beta_{2} E^{2}+\cdots},
$$

where the series on the right-hand side can be easily divided and the resulting coefficients related to the standard scattering length, effective radius, etc.

\section{MULTI-CHANNEL PROBLEM}

For a physical system that can move in $N$ channels, we have a system of $N$ coupled radial Schrödinger equations (the details can be found in Refs. 2 and 4 as well as in Refs. 16, 27, and 28),

$$
\left[\partial_{r}^{2}+k_{n}^{2}-\frac{\ell_{n}\left(\ell_{n}+1\right)}{r^{2}}-\frac{2 k_{n} \eta_{n}}{r}\right] u_{n}(E, r)=\sum_{n^{\prime}=1}^{N} V_{n n^{\prime}}(r) u_{n^{\prime}}(E, r)
$$

where the channel momenta $k_{n}(n=1,2, \ldots, N)$ are given by Eq. (2) and the potentials $V_{n n^{\prime}}(r)$ are assumed to be of a short range. A solution of this system is a column $\left(u_{1}, u_{2}, \ldots, u_{N}\right)^{\mathrm{T}}$, each entry of which describes the motion in an individual channel. There are $2 N$ linearly independent solutions of this kind and only half of them are regular at $r=0 .{ }^{18}$ Combining the regular columns in a square matrix (where the second subscript labels different solutions),

$$
U(E, r)=\left(\begin{array}{cccc}
u_{11} & u_{12} & \ldots & u_{1 N} \\
u_{21} & u_{22} & \ldots & u_{2 N} \\
\vdots & \vdots & \ldots & \vdots \\
u_{N 1} & u_{N 2} & \ldots & u_{N N}
\end{array}\right)
$$

we obtain the so called fundamental matrix of regular solutions (the basis). Any physical solution is a linear combination of its columns.

\section{A. Jost matrix}

At large distances $(r \rightarrow \infty)$, where the right-hand sides of Eq. (53) vanish, they decouple and become pure Coulomb equations. Formally, we can still consider these equations as a system, which has $2 N$ linearly independent solutions. When $r \rightarrow \infty$, there is no requirement of regularity and therefore the full basis consists of all $2 N$ linearly independent column-solutions of the system of Coulomb equations. Of course, the choice of them is not unique. For us, it is convenient to choose either the pair of the diagonal square matrices

$$
\begin{gathered}
F(E, r)=\operatorname{diag}\left\{F_{\ell_{1}}\left(\eta_{1}, k_{1} r\right), F_{\ell_{2}}\left(\eta_{2}, k_{2} r\right), \ldots, F_{\ell_{N}}\left(\eta_{N}, k_{N} r\right)\right\}, \\
G(E, r)=\operatorname{diag}\left\{G_{\ell_{1}}\left(\eta_{1}, k_{1} r\right), G_{\ell_{2}}\left(\eta_{2}, k_{2} r\right), \ldots, G_{\ell_{N}}\left(\eta_{N}, k_{N} r\right)\right\},
\end{gathered}
$$

or their linear combinations

$$
H^{( \pm)}(E, r)=F(E, r) \mp i G(E, r)
$$

which are diagonal matrices of the Coulomb spherical waves (17). 
By analogy with Eq. (7), we look for the fundamental matrix (54) in the form

$$
U(E, r)=F(E, r) A(E, r)+G(E, r) B(E, r)
$$

and, substituting it into the Schrödinger equation (53), obtain the following differential equations for the unknown square matrices $A$ and $B$ :

$$
\begin{aligned}
& \partial_{r} A=K^{-1} G V(F A+G B), \\
& \partial_{r} B=-K^{-1} F V(F A+G B),
\end{aligned}
$$

where $K$ is the diagonal matrix of the channel momenta,

$$
K=\operatorname{diag}\left\{k_{1}, k_{2}, \ldots, k_{N}\right\},
$$

and $V$ is the potential matrix that appears on the right-hand side of Eq. (53). Since $U(E, r)$ is regular at $r=0$, the boundary conditions can be chosen as follows:

$$
A(E, 0)=I, \quad B(E, 0)=0,
$$

which is a direct generalization of the single-channel conditions (10). With the conditions (62), the fundamental matrix behaves near the origin as

$$
U(E, r) \underset{r \rightarrow 0}{\longrightarrow} F(E, r) \underset{r \rightarrow 0}{\longrightarrow} C \operatorname{diag}\left\{\left(k_{1} r\right)^{\ell_{1}+1},\left(k_{2} r\right)^{\ell_{2}+1}, \ldots,\left(k_{N} r\right)^{\ell_{N}+1}\right\},
$$

where $C$ is the diagonal matrix:

$$
C=\operatorname{diag}\left\{C_{\ell_{1}}\left(\eta_{1}\right), C_{\ell_{2}}\left(\eta_{2}\right), \ldots, C_{\ell_{N}}\left(\eta_{N}\right)\right\} .
$$

Similarly to the single-channel case, we consider another fundamental matrix of regular solutions, which differs from $U$ by a constant factor,

$$
\Phi(E, r)=C^{-1} L^{-1} U(E, r) \underset{r \rightarrow 0}{\longrightarrow} \operatorname{diag}\left\{j_{\ell_{1}}\left(k_{1} r\right), j_{\ell_{2}}\left(k_{2} r\right), \ldots, j_{\ell_{N}}\left(k_{N} r\right)\right\},
$$

where we introduced yet another diagonal matrix:

$$
L=\operatorname{diag}\left\{\left(2 \ell_{1}+1\right) ! !,\left(2 \ell_{2}+1\right) ! !, \ldots,\left(2 \ell_{N}+1\right) ! !\right\} .
$$

Matrix $\Phi$ is the one, which is used to define the Jost matrices. Each column of $\Phi$ is a solution of the original system of differential equations (53). Therefore, at large distances it can be written as a linear combination of $2 N$ columns of the basis, i.e., the columns of the matrices $H^{(-)}$and $H^{(+)}$. The combination coefficients can be combined in two square matrices, which gives

$$
\Phi(E, r) \underset{r \rightarrow \infty}{\longrightarrow} H^{(-)}(E, r) \sigma^{(+)} f^{(\text {in })}(E)+H^{(+)}(E, r) \sigma^{(-)} f^{(\text {out })}(E),
$$

with

$$
\sigma^{( \pm)}=\operatorname{diag}\left\{e^{ \pm i \sigma_{\ell_{1}}}, e^{ \pm i \sigma_{\ell_{2}}}, \ldots, e^{ \pm i \sigma_{\ell_{N}}}\right\}
$$

Actually, the Jost matrices $f^{\text {(in/out) }}(E)$ consist of those combination coefficients. Each element of these matrices has two subscripts: one to show which column of $\Phi$ is expanded, and the other to indicate which column of the basis is multiplied and added to the combination. On the other hand, the elements of the Jost matrices can be viewed as the amplitudes of the in-coming and out-going spherical waves for each column of $\Phi$ in each channel.

By analogy with Eq. (20), we introduce the linear combinations of the unknown matrices $A$ and $B$,

$$
\mathcal{F}^{\text {(in/out) }}(E, r)=\frac{1}{2}[A(E, r) \mp i B(E, r)]
$$

which gives

$$
\begin{aligned}
& U(E, r)=H^{(-)}(E, r) \mathcal{F}^{\text {(in) }}(E, r)+H^{(+)}(R, r) \mathcal{F}^{\text {(out) }}(E, r) \\
& \underset{r \rightarrow \infty}{\longrightarrow} H^{(-)}(E, r) \mathcal{F}^{(\text {in })}(E, \infty)+H^{(+)}(E, r) \mathcal{F}^{(\text {out })}(E, \infty),
\end{aligned}
$$


and therefore

$$
f^{(\text {in/out })}(E)=\lim _{r \rightarrow \infty} C^{-1} L^{-1} \sigma^{(\mp)} \mathcal{F}^{(\text {in/out })}(E, r)
$$

\section{B. Analytic structure}

Using the Lambert representation, (25) and (26), we can factorize the diagonal Coulomb matrices (55) and (56) as follows:

$$
\begin{aligned}
& F=D \tilde{F} \\
& G=M D \tilde{F}+K D^{-1} \tilde{G},
\end{aligned}
$$

where

$$
\begin{aligned}
& D=\operatorname{diag}\left\{C_{\ell_{1}}\left(\eta_{1}\right) k_{1}^{\ell_{1}+1}, C_{\ell_{2}}\left(\eta_{2}\right) k_{2}^{\ell_{2}+1}, \ldots, C_{\ell_{N}}\left(\eta_{N}\right) k_{N}^{\ell_{N}+1}\right\}, \\
& M=\operatorname{diag}\left\{\frac{2 \eta_{1} h\left(\eta_{1}\right)}{C_{0}^{2}\left(\eta_{1}\right)}, \frac{2 \eta_{2} h\left(\eta_{2}\right)}{C_{0}^{2}\left(\eta_{2}\right)}, \ldots, \frac{2 \eta_{N} h\left(\eta_{N}\right)}{C_{0}^{2}\left(\eta_{N}\right)}\right\},
\end{aligned}
$$

and the diagonal matrices $\tilde{F}(E, r)$ and $\tilde{G}(E, r)$ are entire functions of $E$.

It should be noted that most of the auxiliary matrices that we introduced are diagonal and therefore commute with each other. This fact is used in further derivations.

Let us transform the unknown matrices $A$ and $B$ as follows:

$$
\begin{aligned}
& \tilde{A}=D A D^{-1}+M D B D^{-1}, \\
& \tilde{B}=K D^{-1} B D^{-1},
\end{aligned}
$$

which generalizes the corresponding single-channel transformation (30) and (31). Using the inverse transformation,

$$
\begin{aligned}
& A=D^{-1} \tilde{A} D-M K^{-1} D \tilde{B} D \\
& B=K^{-1} D \tilde{B} D
\end{aligned}
$$

it is easy to see that

$$
U=F A+G B=(\tilde{F} \tilde{A}+\tilde{G} \tilde{B}) D,
$$

and therefore Eqs. (59) and (60) take the form

$$
\begin{aligned}
& \partial_{r} A=K^{-1} G V(\tilde{F} \tilde{A}+\tilde{G} \tilde{B}) D, \\
& \partial_{r} B=-K^{-1} F V(\tilde{F} \tilde{A}+\tilde{G} \tilde{B}) D .
\end{aligned}
$$

The transformation (76) and (77) shows us how to combine Eqs. (80) and (81) in order to have on the left-hand sides the derivatives $\partial_{r} \tilde{A}$ and $\partial_{r} \tilde{B}$. Multiplying these equations by appropriate operators, we get rid of all the "troublesome" factors (non-holomorphic or causing the branching), and obtain

$$
\begin{aligned}
& \partial_{r} \tilde{A}(E, r)=\tilde{G}(E, r) V(r)[\tilde{F}(E, r) \tilde{A}(E, r)+\tilde{G}(E, r) \tilde{B}(E, r)], \\
& \partial_{r} \tilde{B}(E, r)=-\tilde{F}(E, r) V(r)[\tilde{F}(E, r) \tilde{A}(E, r)+\tilde{G}(E, r) \tilde{B}(E, r)],
\end{aligned}
$$

which is the matrix generalization of the single-channel equations (34) and (35). The boundary conditions,

$$
\tilde{A}(E, 0)=I, \quad \tilde{B}(E, 0)=0,
$$


remain simple and energy-independent. Therefore, in the same way as we did it in the single-channel case, we can use the Poincaré theorem. Actually, everything what was said in Sec. II B about the single-channel functions $\tilde{A}_{\ell}(E, \infty)$ and $\tilde{B}_{\ell}(E, \infty)$ remains valid for the matrices $\tilde{A}(E, \infty)$ and $\tilde{B}(E, \infty)$. They are analytic single-valued functions of $E$ within the domain $\mathcal{D}$ along the real positive axis.

Therefore, we have established that within the domain $\mathcal{D}$, the multi-channel Jost matrices, for a combination of the Coulomb and short-range interactions, have the following analytic structure:

$$
f^{\text {(in/out) }}=\frac{1}{2} C^{-1} L^{-1} \sigma^{(\mp)}\left[D^{-1} \tilde{A} D-(M \pm i) K^{-1} D \tilde{B} D\right]
$$

In the explicit form for their matrix elements this reads $(m, n=1,2, \ldots, N)$ :

$$
\begin{aligned}
f_{m n}^{(\text {in } / \text { out })}(E) & =\frac{e^{\pi \eta_{m} / 2} \ell_{m} !}{2 \Gamma\left(\ell_{m}+1 \pm i \eta_{m}\right)}\left\{\frac{C_{\ell_{n}}\left(\eta_{n}\right) k_{n}^{\ell_{n}+1}}{C_{\ell_{m}}\left(\eta_{m}\right) k_{m}^{\ell_{m}+1}} \tilde{A}_{m n}(E, \infty)-\right. \\
& \left.-\left[\frac{2 \eta_{m} h\left(\eta_{m}\right)}{C_{0}^{2}\left(\eta_{m}\right)} \pm i\right] C_{\ell_{m}}\left(\eta_{m}\right) C_{\ell_{n}}\left(\eta_{n}\right) k_{m}^{\ell_{m}} k_{n}^{\ell_{n}+1} \tilde{B}_{m n}(E, \infty)\right\} .
\end{aligned}
$$

The $S$-matrix can be obtained by calculating the product (3). It is easy to check that for neutral particles $(\eta \rightarrow 0)$, we come back to the structure given by Eq. (4).

\section{Attractive Coulomb forces}

Consider the case when the multi-channel potential involves (at least in one channel) a combination of a short-range and an attractive Coulomb forces. In that particular channel (or channels) the pure Coulomb part of the potential generates the hydrogen-like spectrum of bound states (38). If there are several channels with attractive Coulomb forces, their pure Coulombic spectral points, generally speaking, do not coincide because the corresponding binding energies are counted from different thresholds and the charges as well as the reduced masses are usually different in different channels. An example, when the Coulomb attraction is present in the third and the fourth channels, is schematically shown in Fig. 3. Of course some coincidences may happen (when the channel thresholds are too close to each other), but they do not affect the reasoning given below.

If the complex variable $E$ is not close to one of the pure Coulombic spectral points, the factorized representations (86) can be used as they are, without any difficulties. If, however, the energy approaches one of these spectral points, the representations (86) have the same $0 \times \infty$ uncertainty as is discussed in Sec. II B 1 and in the Appendix.

Suppose the energy $E$ is close to a Coulombic spectral point in the channel $j$. If the Coulombic spectral points in different channels do not coincide, then only one row $(n=j)$ and one column $(m=j)$ of the matrices (86) are affected. These row and column are always crossed at the diagonal $(n=m=j)$.

The diagonal element of (86) has exactly the same structure as the single-channel Jost function (40), and thus the uncertainty $0 \times \infty$ for it is resolved in the same way. For the off-diagonal elements

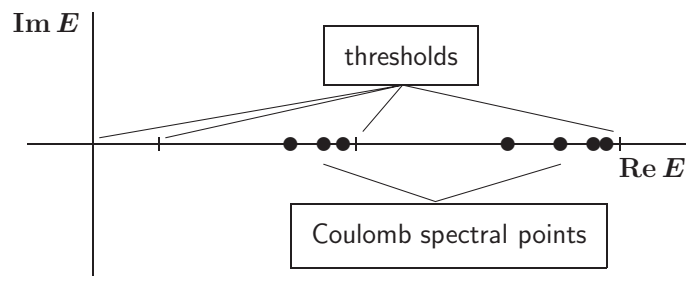

FIG. 3. Schematically shown pure Coulomb spectral points in the third and fourth channels (below the corresponding thresholds). 
of the row (when the singularities are associated with the left-hand side subscript), we have

$$
f_{m n}^{\text {(in/out) }}\left(E_{n}^{c}+\delta\right) \underset{\delta \rightarrow 0}{\longrightarrow} \frac{\text { const }}{1 / \delta}\left(\frac{\text { const }}{\sqrt{1 / \delta}}-\frac{\text { const }}{1 / \delta} \sqrt{1 / \delta}\right) \underset{\delta \rightarrow 0}{\longrightarrow} 0,
$$

where $\delta$ is an arbitrary complex number that shifts the energy from the spectral point, and the leading terms of various quantities involved in (86) are taken from Eqs. (A2), (A3), (A6), (A7), and (A13) of the Appendix.

For the off-diagonal elements of the column, the only troublesome quantity is the Coulomb penetration factor $C_{\ell_{n}}\left(\eta_{n}\right)$, and therefore,

$$
\begin{aligned}
f_{m n}^{\text {(in/out })}\left(E_{n}^{c}+\delta\right) \underset{\delta \rightarrow 0}{\longrightarrow} & \frac{e^{\pi \eta_{m} / 2} \ell_{m} !}{2 \Gamma\left(\ell_{m}+1 \pm i \eta_{m}\right)}\left\{\frac{k_{n}^{\ell_{n}+1}}{C_{\ell_{m}}\left(\eta_{m}\right) k_{m}^{\ell_{m}+1}} \tilde{A}_{m n}-\right. \\
& \left.-\left[\frac{2 \eta_{m} h\left(\eta_{m}\right)}{C_{0}^{2}\left(\eta_{m}\right)} \pm i\right] C_{\ell_{m}}\left(\eta_{m}\right) k_{m}^{\ell_{m}} k_{n}^{\ell_{n}+1} \tilde{B}_{m n}\right\} \times \frac{\text { const }}{\sqrt{\delta}} .
\end{aligned}
$$

It looks like these elements diverge. However, since the Jost matrices are non-singular, we conclude that the expression in the curly brackets tends to zero as $\sqrt{\delta}$ or faster, when $\delta \rightarrow 0$.

\section{Power-series expansions}

Since $\tilde{A}(E, r)$ and $\tilde{B}(E, r)$ are holomorphic (for $r=\infty$, within the domain $\mathcal{D}$ ), they can be expanded in the Taylor series

$$
\begin{aligned}
& \tilde{A}(E, r)=\sum_{n=0}^{\infty} \alpha_{n}\left(E_{0}, r\right)\left(E-E_{0}\right)^{n}, \\
& \tilde{B}(E, r)=\sum_{n=0}^{\infty} \beta_{n}\left(E_{0}, r\right)\left(E-E_{0}\right)^{n},
\end{aligned}
$$

near and arbitrary point $E_{0} \in \mathcal{D}$. The expansion coefficients $\alpha_{n}$ and $\beta_{n}$ are $(N \times N)$-matrices.

Similarly to Eqs. (44) and (45), the holomorphic parts of the matrices $F$ and $G$ can also be expanded in the Taylor series around the same point $E_{0}$,

$$
\begin{aligned}
& \tilde{F}(E, r)=\sum_{n=0}^{\infty} \varphi_{n}\left(E_{0}, r\right)\left(E-E_{0}\right)^{n}, \\
& \tilde{G}(E, r)=\sum_{n=0}^{\infty} \gamma_{n}\left(E_{0}, r\right)\left(E-E_{0}\right)^{n},
\end{aligned}
$$

where $\varphi_{n}$ and $\gamma_{n}$ are also matrices. In exactly the same way as it was done in the single-channel case, we obtain the same system of differential equations (46) and (47) with the boundary conditions:

$$
\alpha_{n}\left(E_{0}, 0\right)=\delta_{n 0} I, \quad \beta_{n}\left(E_{0}, 0\right)=0, \quad \forall n, E_{0} .
$$

Of course finding the expansion coefficients $\alpha_{n}$ and $\beta_{n}$ for an arbitrary central point $E_{0}$ is not an easy task. The main difficulty is that to solve Eqs. (46) and (47), we need the corresponding expansion coefficients $\varphi_{n}$ and $\gamma_{n}$ of the Coulomb functions. In principle, these coefficients can be found via the derivatives $\partial^{n} / \partial E^{n}$ of the Coulomb functions at $E=E_{0}$. We are aware of at least one well-developed procedure for calculating these derivatives at $E_{0}=0$, namely, the technique used in Ref. 29. For an arbitrary $E_{0}$, an additional work is needed to develop such a technique, based, for example, on the explicit expressions for the functions $\tilde{F}_{\ell}(E, r)$ and $\tilde{G}_{\ell}(E, r)$ (see Refs. 22 and 23).

As we mentioned before, instead of finding $\alpha_{n}$ and $\beta_{n}$ by solving Eqs. (46) and (47), we can obtain them from experimental data as fitting parameters. Taking few terms in Eqs. (87) and (88) and substituting them in (86) and then in (3), we can fit any data (cross section, polarization, etc.) available near the energy $E_{0}$. After such a fit, the resulting $S$-matrix can be used for locating the nearby resonances or for calculating the observables for which no data are available. 
When doing a fitting procedure it is important to keep the number of the varying parameters to a minimum. Although such a procedure does not involve solving Eqs. (46) and (47), the fact that $\alpha_{n}$ and $\beta_{n}$ obey these equations may help us to reduce the number of fitting parameters. Indeed, if $E_{0}$ is real then everything in these equations as well as the boundary conditions is real, which implies that $\alpha_{n}$ and $\beta_{n}$ are also real. If however $E_{0}$ is taken in the complex plane, then the number of fitting parameters is doubled because $\alpha_{n}$ and $\beta_{n}$ become complex.

\section{CONCLUSION}

Our present work is based on the representation of the standard Coulomb functions suggested by Lambert, ${ }^{21}$ who separated them in the holomorphic parts and non-holomorphic factors. Our main task was to find a similar factorization of the multi-channel Jost matrices for a quantum system whose inter-particle interaction involves both the short-range and Coulomb potentials.

In order to achieve this, we first transformed the multi-channel radial Schrödinger equation into an equivalent system of first-order differential equations for the matrix functions that asymptotically tend to the Jost matrices. Then we factorized these matrix functions in such a way that all the nonholomorphic factors in the equations canceled out. The remaining differential equations together with the energy-independent boundary conditions define holomorphic functions of the energy.

Our main result is the representation (86) of the Jost matrices, where $\tilde{A}$ and $\tilde{B}$ are holomorphic functions of $E$, while all the other factors are responsible for the branching of the Riemann surface. When $\tilde{A}$ and $\tilde{B}$ are found (either in exact or approximate way) the Jost matrices, and via them the $S$-matrix, become available on all the sheet of the Riemann surface. The proper topology of this surface is always guaranteed, no matter how accurate the matrices $\tilde{A}$ and $\tilde{B}$ are.

Being holomorphic, the matrices $\tilde{A}$ and $\tilde{B}$ can be expanded in the Taylor series near practically an arbitrary point $E_{0}$ where it could be interesting from the point of view of physical applications. This is a generalization of the effective-range expansion. However, in our case such an expansion is not restricted to the near-threshold energies.

We derived a set of differential equations, whose solutions give the expansion coefficients. Alternatively, instead of calculating them, these coefficients can be used as the free parameters to fit the scattering data available around a chosen energy $E_{0}$. After finding an optimal set of such parameters, the approximate matrices $\tilde{A}$ and $\tilde{B}$ substituted in (86) give the Jost matrices with proper analytic structure and valid within certain circle around $E_{0}$ on all the sheets of the Riemann surface. This would enable one to extract from experimental data the parameters of possible resonances as zeros of $\operatorname{det} f^{(\mathrm{in})}(E)$ at complex energies. In Ref. 17, where we used the representation (4), it was demonstrated that such a procedure is efficient and accurate for the case of a short-range potential.

\section{ACKNOWLEDGMENTS}

We are grateful for the support from the Carl Trygger and the Magnus Bergvall foundations as well as from the National Research Foundation of South Africa.

\section{APPENDIX: JOST FUNCTION NEAR THE COULOMB SPECTRAL POINTS}

If the Coulombic part of the potential is attractive, the factorized representation (37) for the Jost function $f_{\ell}^{\text {(in) }}(E)$ needs special treatment when $E$ is close to any of the Coulombic spectral points $E_{n}^{c}$ given by Eq. (38). Indeed, in such a case the Sommerfeld parameter becomes pure imaginary, $\eta=i n, n=1,2,3, \ldots$, which makes singular the $\Gamma$-function as well as $h(\eta)$ and $C_{\ell}(\eta)$ in the expression

$$
f_{\ell}^{(\mathrm{in})}(E)=\frac{e^{\pi \eta / 2}(2 \ell) ! !}{2^{\ell+1} \Gamma(\ell+1+i \eta)}\left\{\tilde{A}_{\ell}-\left[2 \eta h(\eta)+i C_{0}^{2}(\eta)\right] \frac{C_{\ell}^{2}(\eta)}{C_{0}^{2}(\eta)} k^{2 \ell+1} \tilde{B}_{\ell}\right\} .
$$

It should be noted that the functions $\tilde{A}_{\ell}(E, \infty)$ and $\tilde{B}_{\ell}(E, \infty)$ are finite and in general are non-zero at $E_{n}^{c}$. In order to find out if and how the singular quantities cancel each other, we need to obtain at 
least the leading terms of their behaviour when the energy approaches $E_{n}^{c}$, i.e., when

$$
E=E_{n}^{c}+\delta, \quad \delta \rightarrow 0,
$$

where $\delta$ is an arbitrary complex number. This energy corresponds to

$$
k=\sqrt{\frac{2 \mu\left(E_{n}^{c}+\delta\right)}{\hbar^{2}}} \approx k_{n}+\frac{k_{n} \delta}{2 E_{n}^{c}}
$$

and

$$
\eta=\frac{\mu e^{2} Z_{1} Z_{2}}{\hbar^{2} k_{n}\left(1+\frac{\delta}{2 E_{n}^{c}}\right)} \approx \eta_{n}\left(1-\frac{\delta}{2 E_{n}^{c}}\right)=i(n+\epsilon), \quad \epsilon \rightarrow 0
$$

where

$$
\epsilon=-\frac{n}{2 E_{n}^{c}} \delta
$$

For the $\Gamma$-function, this gives

$$
\Gamma(\ell+1+i \eta)=\Gamma\left(\ell+1-n_{r}-\ell-1-\epsilon\right)=\Gamma\left(-n_{r}-\epsilon\right) .
$$

In order to get rid of the negative integer, $-n_{r}$, in this $\Gamma$-function, the recurrence relation (Eq. (6.1.15) of Ref. 30)

$$
\Gamma(z)=\frac{1}{z} \Gamma(z+1)
$$

is applied $n_{r}$ times. This gives

$$
\begin{aligned}
\Gamma\left(-n_{r}-\epsilon\right) & =\frac{1}{-n_{r}-\epsilon} \Gamma\left(1-n_{r}-\epsilon\right)=\frac{1}{\left(-n_{r}-\epsilon\right)\left(1-n_{r}-\epsilon\right)} \Gamma\left(2-n_{r}-\epsilon\right)=\cdots \\
& =\frac{1}{\left(-n_{r}-\epsilon\right)\left(1-n_{r}-\epsilon\right) \cdots\left(n_{r}-n_{r}-\epsilon\right)} \Gamma\left(n_{r}+1-n_{r}-\epsilon\right) \\
& =\frac{(-1)^{n_{r}+1}}{n_{r} !} \cdot \frac{1}{\epsilon} .
\end{aligned}
$$

Therefore, the pure Coulomb Jost function linearly vanishes with $\epsilon$ at each point $E_{n}^{c}$.

Consider the other singular quantity in Eq. (A1), namely, the product

$$
2 \eta h(\eta)=\eta[\psi(i \eta)+\psi(-i \eta)]-2 \eta \ln \hat{\eta},
$$

where the Digamma function $\psi(z)$ is singular when $z$ is a non-positive integer (see Ref. 30). The only quantity in (A4) that becomes singular at a Coulomb spectral point is

$$
\psi(i \eta)=\psi(-n-\epsilon) .
$$

To find out how fast it goes to infinity with $\epsilon \rightarrow 0$, we apply $(n+1)$ times the recurrence relation (see Eq. (6.3.5) of Ref. 30)

$$
\psi(z)=\psi(z+1)-\frac{1}{z} .
$$

As a result, we increment the argument of the function (A5) until it becomes positive:

$$
\begin{aligned}
\psi(-n-\epsilon) & =\psi(1-n-\epsilon)-\frac{1}{-n-\epsilon}=\psi(2-n-\epsilon)-\frac{1}{1-n-\epsilon}-\frac{1}{-n-\epsilon}=\ldots \\
& =\psi(1-\epsilon)-\frac{1}{-\epsilon}-\frac{1}{-1}-\frac{1}{-2}-\cdots-\frac{1}{-n} .
\end{aligned}
$$

Since $\psi(1)=-\gamma=-0.5772156649 \ldots$ (Euler's constant), we obtain

$$
\psi(-n-\epsilon)=\frac{1}{\epsilon}-\gamma+\left(1+\frac{1}{2}+\frac{1}{3}+\cdots+\frac{1}{n}\right),
$$


and thus

$$
2 \eta h(\eta) \underset{\epsilon \rightarrow 0}{\longrightarrow} \frac{i n}{\epsilon}
$$

Consider now the behaviour of

$$
C_{0}^{2}(\eta)=\frac{2 \pi \eta}{e^{2 \pi \eta}-1}
$$

near a Coulomb spectral point. With $\eta=i(n+\epsilon)$, we have

$$
\begin{aligned}
C_{0}^{2}(i n+i \epsilon) & =\frac{2 \pi i n}{e^{2 \pi i n} e^{2 \pi i \epsilon}-1}=\frac{2 \pi i n}{e^{2 \pi i \epsilon}-1} \\
& =\frac{2 \pi i n}{1+2 \pi i \epsilon+\frac{1}{2}(2 \pi i \epsilon)^{2}+\cdots-1} \underset{\epsilon \rightarrow 0}{\longrightarrow} \frac{n}{\epsilon},
\end{aligned}
$$

and thus

$$
2 \eta h(\eta)+i C_{0}^{2}(\eta) \underset{\epsilon \rightarrow 0}{\longrightarrow} \frac{2 i n}{\epsilon}
$$

What remains now is to analyze the behaviour of the ratio $C_{\ell}^{2}(\eta) / C_{0}^{2}(\eta)$ near the Coulomb spectral points. For this purpose, we can use the relations (14.1.9) and (14.1.11) of Ref. 30,

$$
\frac{C_{\ell}^{2}(\eta)}{C_{0}^{2}(\eta)}=\frac{\left(1+\eta^{2}\right)\left(4+\eta^{2}\right) \cdots\left(\ell^{2}+\eta^{2}\right) 2^{2 \ell}}{(2 \ell+1)^{2}[(2 \ell) !]^{2}}, \quad \ell \geqslant 1 .
$$

Looking at

$$
\ell^{2}+\eta^{2}=\ell^{2}-n^{2}=\ell^{2}-\left(n_{r}+\ell+1\right)^{2}=-\left[\left(n_{r}+1\right)^{2}+2 \ell\left(n_{r}+1\right)\right]<0,
$$

we see that none of the factors in the numerator of Eq. (A9) can be zero. And apparently the ratio (A9) is finite:

$$
\frac{C_{\ell}^{2}(-i n-i \epsilon)}{C_{0}^{2}(-i n-i \epsilon)} \underset{\epsilon \rightarrow 0}{\longrightarrow} \text { const }\left\{\begin{array}{l}
\neq 0 \\
\neq \infty
\end{array} .\right.
$$

Substituting (A3) and (A8) into Eq. (A1), we obtain

$$
\begin{aligned}
f_{\ell}^{(\mathrm{in})} & \underset{\epsilon \rightarrow 0}{\longrightarrow} \frac{i^{n}(2 \ell) ! ! n_{r} !}{2^{\ell+1}(-1)^{n_{r}+1}}\left(\epsilon \tilde{A}_{\ell}-2 i n \cdot \frac{C_{\ell}^{2}}{C_{0}^{2}} k_{n}^{2 \ell+1} \tilde{B}_{\ell}\right) \\
& \underset{\epsilon \rightarrow 0}{\longrightarrow} \frac{i^{n+1}(2 \ell) ! ! n_{r} ! n}{2^{\ell}(-1)^{n_{r}}} \cdot \frac{C_{\ell}^{2}}{C_{0}^{2}} k_{n}^{2 \ell+1} \tilde{B}_{\ell} .
\end{aligned}
$$

This equation shows that in general

$$
f_{\ell}^{(\mathrm{in})}\left(E_{n}^{c}\right) \neq 0
$$

i.e., (as it should be) the Coulomb spectral points are not the spectral points anymore when a shortrange potential is added. Moreover, the singularities exactly cancel each other and the Jost function $f_{\ell}^{(i n)}$ is regular at $E_{n}^{c}$. Of course it may happen that $\tilde{B}_{\ell}\left(E_{n}^{c}, \infty\right)=0$ and then the corresponding point is a spectral point of the total potential. This, however, would be just a coincidence. As far as $f_{\ell}^{\text {(out) }}$ is concerned, it is also regular at $E_{n}^{c}$ because it involves the difference $2 \eta h(\eta)-i C_{0}^{2}(\eta)$, where the singularities $\sim 1 / \epsilon$ exactly cancel each other as well.

For doing a similar analysis of the multi-channel Jost matrices, it is useful to note that

$$
C_{\ell}(-i n-i \epsilon) \underset{\epsilon \rightarrow 0}{\longrightarrow} \frac{\text { const }}{\sqrt{\epsilon}},
$$

which follows from Eqs. (A10) and (A7). The constant can be found using Eq. (A9). 
${ }^{1}$ G. F. Chew, The Analytic S-Matrix (W. A. Benjamin, Inc., New York, 1966).

${ }^{2}$ J. R. Taylor, Scattering Theory (John Wiley and Sons, New York, 1972).

${ }^{3}$ S. A. Rakityansky and N. Elander, "Generalized effective-range expansion,” J. Phys. A 42, 225302 (2009).

${ }^{4}$ A. M. Badalyan, L. P. Kok, M. I. Polikarpov, and Yu. A. Simonov, "Resonances in coupled channels in nuclear and particle physics," Phys. Rep. 82(2), 31 (1982).

${ }^{5}$ M. H. Ross and G. L. Shaw, "Scattering length and effective range theory for multi-channel processes," Ann. Phys. 9, 391-415 (1960).

${ }^{6}$ M. H. Ross and G. L. Shaw, "Multichannel effective range theory," Ann. Phys. 13, 147-186 (1961).

${ }^{7}$ P. Nath and G. L. Shaw, "Multichannel effective-range theory from the N/D formalism," Phys. Rev. 138(3B), B702 (1965).

${ }^{8}$ M. W. Kermode, "Effective range theory for multi-channel scattering," Nucl. Phys. A 99, 605-624 (1967).

${ }^{9}$ D. W. L. Sprung, M. W. Kermode, and S. Klarsfeld, "On the application of effective-range theory to the ${ }^{3} S_{1}-{ }^{3} D_{1}$ state of the neutron-proton system," J. Phys. G 8, 923-935 (1982).

${ }^{10}$ L. Rosenberg, "Multichannel effective-range theory with long-range interactions," Phys. Rev. A 57(3), 1862-1869 (1998).

${ }^{11}$ R. H. Dalitz and S. Tuan, "The phenomenological representation of $K$-nucleon scattering and reaction amplitudes," Ann. Phys. (N.Y.) 10, 307-351 (1960).

${ }^{12}$ V. I. Kukulin, V. M. Krasnopolsky, and J. Horáček, Theory of Resonances (Kluwer Academic Publishers, Dordrecht, 1989).

${ }^{13}$ Yu. S. Surovtsev et al., "Parameters of scalar resonances from the combined analysis of data on processes $\pi \pi \rightarrow \pi \pi, K \bar{K}, \eta \eta$ and $J / \psi$ decays," preprint arXiv:1207.6937v1 [hep-ph] (2012).

${ }^{14}$ K. Willner and F. A. Gianturco, "Low-energy expansion of the Jost function for long-range potentials," Phys. Rev. A 74, 052715 (2006).

${ }^{15}$ V. V. Pupyshev, "Perturbation theory for the one-dimensional Schrödinger scattering problem," J. Phys. A 28, 3305 (1995).

${ }^{16}$ S. A. Rakityansky and N. Elander, "Multi-channel analog of the effective-range expansion," J. Phys. A 44, 115303 (2011).

${ }^{17}$ S. A. Rakityansky and N. Elander, "A method for extracting the resonance parameters from experimental cross sections," Int. J. Mod. Phys. E 22, 1350032 (2013).

${ }^{18}$ L. Brand, Differential and Difference Equations (Wiley, New York, 1966).

${ }^{19}$ S. A. Rakityansky, S. A. Sofianos, and K. Amos, "A method for calculating the Jost function for analytic potentials," Nuovo Cimento B 111, 363-378 (1996).

${ }^{20}$ S. A. Sofianos and S. A. Rakityansky, "Exact method for locating potential resonances and Regge trajectories," J. Phys. A 30, 3725 (1997).

${ }^{21}$ E. Lambert, "Fonction de portée effective et déplacement en énergie des états liés en présence d'un potentiel coulombien modifié," Helv. Phys. Acta 42, 667-677 (1969).

${ }^{22}$ D. Bollé and F. Gesztesy, "Scattering observables in arbitrary dimension $n \geq 2$," Phys. Rev. A 30, 1279 (1984).

${ }^{23}$ S. König, D. Lee, and H.-W. Hammer, "Causality constraints for charged particles," J. Phys. G: Nucl. Part. Phys. 40, 045106 (2013).

${ }^{24}$ H. Poincaré, "Sur les groupes des équations linéaires," Acta Math. 4, 201-311 (1884).

${ }^{25}$ S. Lefschetz, Differential Equations: Geometric Theory (Interscience Publishers, New York, 1957).

${ }^{26}$ S. A. Rakityansky and N. Elander, "Analytic structure and power series expansion of the Jost function for the twodimensional problem," J. Phys. A 45, 135209 (2012).

${ }^{27}$ S. A. Rakityansky and S. A. Sofianos, "Jost function for coupled partial waves," J. Phys. A 31, 5149-5175 (1998).

${ }^{28}$ S. A. Rakityansky and N. Elander, "Analyzing the contribution of individual resonance poles of the $S$-matrix to the two-channel scattering," Int. J. Quantum Chem. 106, 1105 (2006).

${ }^{29}$ O. L. R. Suárez and J.-M. Sparenberg, "Precise determination of the effective-range parameters up to an arbitrary order," Phys. Rev. C 88, 014601 (2013).

${ }^{30}$ Handbook of Mathematical Functions, edited by M. Abramowitz and I. A. Stegun (Dover Publications, Inc., New York, 1972). 